\title{
JIMS Extensions for Resource Monitoring and Management of Solaris 10
}

\author{
Krzysztof Zieliński, Marcin Jarząb, Damian Wieczorek, and Kazimierz Balos \\ Institute of Computer Science, University of Science and Technology, \\ Al. Mickiewicza 30, 30-059 Krakow, Poland \\ $\{\mathrm{kz}, \mathrm{mj}, \mathrm{dwieczor}, \mathrm{kbalos}\}$ agh.edu.pl \\ http://www.ics.agh.edu.pl
}

\begin{abstract}
This paper describes extensions of the JIMS system with selected mechanisms of Solaris 10 resources monitoring and management. The proposed extensions together with JIMS functionality create a foundation for the adaptive layer implementation of large distributed computer systems. The proposed solution addresses a heterogeneous self-configuring execution environment typical of Grids system. The paper presents not only the innovative concept of the proposed extensions but their existing implementation.
\end{abstract}

\section{Introduction}

Resource monitoring and management is one of most sought-after aspects of functionality of large computer systems, supporting their adaptability to changing workload and end-user requirements. Modern operating systems, like Solaris10, offer very rich and complex mechanisms of resource control. Being a part of large heterogeneous distributed systems, i.e. Grids, the computers running Solaris10 should expose their resource management mechanisms regarding projects and zones $[10,11]$ in a uniform way so as to be accessible from the integrated management platform.

This paper describes an extension of the JIMS [1,2] platform with the concepts of Solaris10 projects and zones monitoring and management. The proposed extension has been implemented using JMX [3, 4] and expands the rich functionality of JIMS from Linux and Solaris 8, 9 to the most advanced features of Solaris10. The proposed extensions make these features accessible to applications written in Java and open a wide space for adaptive policy-driven software layer implementation. These extensions, integrated with JIMS, create a very advanced and powerful tool, beyond the capabilities of comparable existing solutions.

The structure of the paper is as follows. In Section 2 a very brief description of JIMS is presented. Next, in Section 3, the Solaris10 resource management mechanisms exploited in this work are summarized. The idea of JIMS extensions and exposition of requested mechanisms is specified in Section 4. The implementation of this extension as JIMS MBeans is presented in Section 5. The paper finishes with conclusions. 


\section{JIMS Overview}

JIMS - the JMX-based Infrastructure Monitoring System assumes that monitored resources are represented as MBeans (Managed Beans), the simple Java objects installed in MBean Servers, key components of JMX (Java Management Extensions). The main purpose of the JIMS is to provide an integrated infrastructure (computational nodes, memory usage, network utilization) and application (Grid engines, J2EE application servers) monitoring management platform. Its features, such as adaptation to operating systems, kernels, and IP protocols, auto-configuration facility (cluster level auto-configuration, Grid level auto-configuration), and dynamic deployment of proper monitoring sensors from one common modules repository, makes it well suited for Grid environments. The JIMS communication model depicted in Fig. 1 can be split into a four-layer architecture:

- The Instrumentation Layer provides infrastructure and Java application monitoring information using specially-designed sensor modules installed in the JIMS Monitoring Agent (JIMS MA).

- The Interoperability Layer provides a common point of communication with computational nodes (also called Worker Nodes or WNs) in clusters through dedicated access nodes (Access Node -AN).

- The Integration Layer enables discovery of all accessible clusters and provides an overall view of the Grid. This layer consists of Global Discovery modules installed in chosen JIMS agents running on Access Nodes.

- The Client Application Layer consists of applications connected to the JIMS system, which are consumers of the information produced by the system.

The extensions proposed in this paper cover the instrumentation layer. The layer is equipped with Mbeans for Solaris 10 projects and zones monitoring and management.

JIMS has been deployed as a monitoring infrastructure at many Grid installations, including the EU IST CrossGrid project [1], the Polish national project Clusterix, and

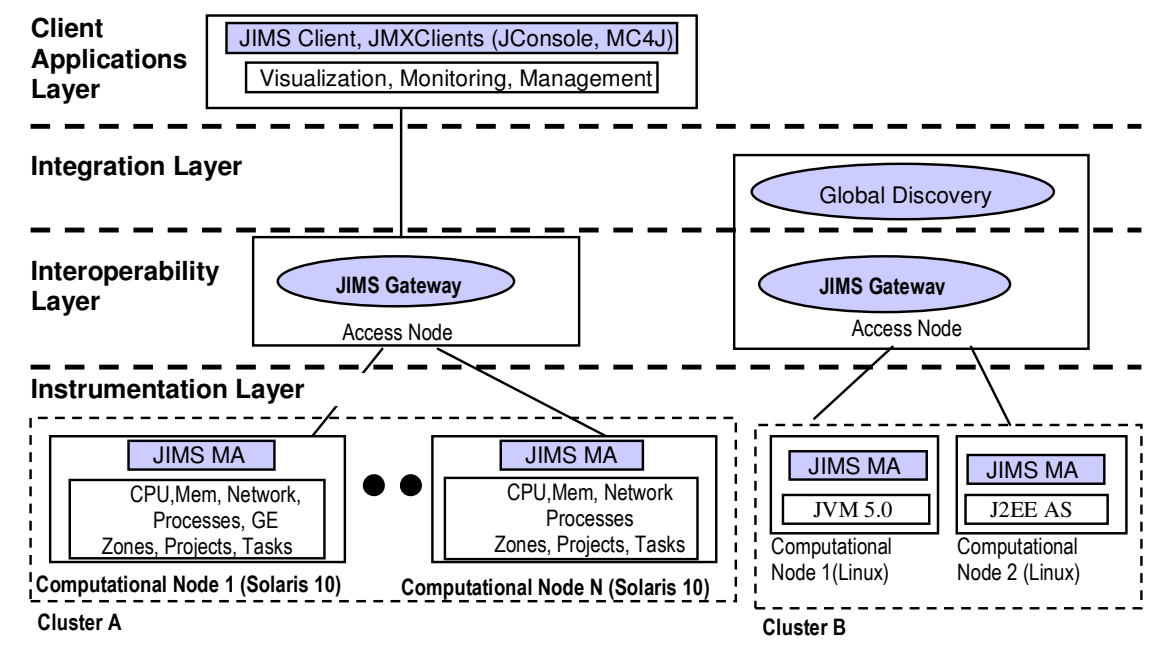

Fig. 1. Layered architecture of communication between JIMS modules 
the internal installation of the N1 Sun Fire 1600 Blade System at the Department of Computer Science AGH/UST in Kraków. These JIMS applications show that features such as hierarchical information representation, fast response time ( 200[ms]), scalable architecture, open source code (AXIS, JMX, SNMP, MX4J), WS interface and JSR003, 160, 255 compatibility, allow for constructing services which are easy to administer and maintain, and which allow easy integration with other components of the Grid. This is why JIMS was selected for proposed extensions regarding Solaris 10 resource monitoring and management.

\section{Resource Monitoring and Management of Solaris 10 Resources}

Solaris $10[7,8,9,10,11]$ provides control resource usage based on workloads. A workload is an aggregation of all processes of an application, or group of applications, which makes sense from a business perspective. The Solaris OS provides a facility called projects to identify workloads. The project serves as an administrative tag used for group-related work in a manner deemed useful by the system administrator.

Once workloads are identified and labeled using projects, the next step in managing resource usage involves measuring workload resource consumption. Current consumption can be measured using the prstat command to obtain real-time snapshot of resource usage. This command reads data stored in /proc VFS (Virtual File System), where each process in the zone has its own subdirectory (in the global zone there are also entries for processes from local zones). The most important information is contained in psinfo, usage and status files. By summarizing information about resource usage of processes it's possible to calculate resource usage of projects and zones. The capability to look at historical data is provided by the Extended Accounting facility which allows collection of statistics at the process level, task level or both. A task is a group of related processes executing in the same project.

Resource controls are configured through the project database. The last field of the project entry is used to set resource controls. The resource controls can be administered by two commands: prctl (get or set resource controls on a running process, task or project) and rctladm (display or modify the global state of system resource controls). This state is stored in the /etc/project file whose changes can be monitored directly. Resource controls can define actions which should be performed when resource usage is crosses some predetermined upper bound. These actions can be observed through messages in the /var/admin/messages log file or catching sent signals to those processes.

Solaris Zones provide a means to create one or more virtual environments on a single operating system instance, shielding applications from details of the underlying hardware. Applications in a single zone run in isolation from applications in other zones. They cannot see, monitor or affect processes running in another zone. The exception is the global zone which encompasses the entire system and is comparable to a normal Solaris OS instance. It has access to the physical hardware and can see and control all processes. The administrator of the global zone can control the system as a whole. The global zone always exists, even when no other zones are configured. Inside the global zone are local zones. These zones are isolated from the physical hardware characteristics of the machine by the virtual platform layer. This layer 
provides zones with a virtual network interface, one or more file systems and a virtual console. The virtual console can be accessed from the global zone using the zlogin command.

Zone administration tasks can be divided into two parts, global zone administration tasks such as creating a zone, and local zone administration tasks such as performing configuration within a zone. The four primary global zone administration tasks are: configuration, installation, virtual platform management, zone login. The configuration task defines zone resource controls which are enabled when the zone is booted.

Regular resource management facilities such as resource controls, projects, and more are available inside zones. Because projects are also virtualized inside a zone, each zone has its own project database. This allows a local zone administrator to configure projects and resource controls local to that zone. Furthermore, the Extended Accounting framework has been extended for zones. Each zone has its own extended accounting files for task-and process-based accounting that contain accounting records exclusively for that zone.

\section{JIMS Extension Concept}

The presented advanced resource management techniques provided by the Solaris 10 introduce a number of logical elements that must be effectively managed and configured. Such a system must be able to collect and publish various kinds of information about configured elements on many hardware virtualized nodes. The main assignments to be performed by the proposed JIMS extensions are:

- Collecting and publishing monitoring information about zones, projects and tasks; the system must be able to expose real-time information about the current resource usage e.g. CPU, memory, resource controls. Because Solaris 10 also introduces Extended Accounting facilities, such information might be stored in a database installed on the JIMS Access Node responsible for the management of Grid Computational Nodes. This will enable e.g. billing the customers for consumed resources or even calculating long-time resource usage trends. Since the system administrator may perform some operations using native system tools, the Monitoring Agent must also reflect these changes.

- Management of zones; adding or removing a given zone, lifecycle-related operations (shutdown, halt, boot, ready), modifying zone values (devices, file systems, attributes, resource controls).

- Management of projects; adding or removing projects, also modifying existing projects' attributes (name, comment, assigned users and groups, resource controls).

Non-functional requirements include ease of use and security. The first requirement implies that running various system components should be relatively simple (minimal need for human interaction) and also installation and configuration of JIMS on many Computing Nodes should be automatic. This may be performed by using N1 Service Provisioning Systems (N1 SPS) [5] which can install JIMS on many nodes. Automatic installation of modules containing MBeans for monitoring and management is performed by JIMS using the JMX Mlet-Service. 
The security aspect must take into account many things related to a broad variety of managed resources which means that some operations such as creating zones, modifying the projects database etc. may be allowed only for restricted groups of users. The privileged position of operations performed in the global zone should be very carefully analyzed. It is necessary to take into account situations when a security domain consists only of local zones distributed over different global zones (physical computers). An example of such a case is represented by Cluster B in Fig. 2.

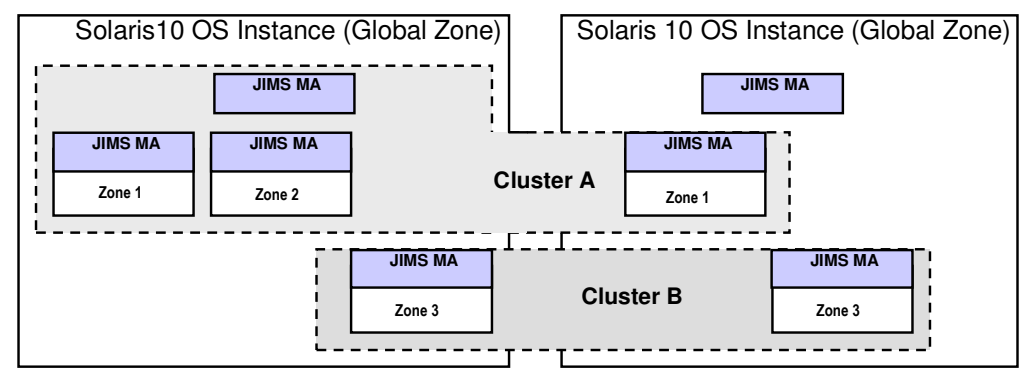

Fig. 2. Intersected clusters which consist of zones installed on different Solaris 10 instances

This is why two deployment scenarios: Global Access and Restricted Access, have to be considered. Clients in the Global Access scenario (see Fig. 3a) connect only to JIMS MA installed in the global zone which has permission to perform operations on all local zones in the current Solaris 10 OS instance. In the Restricted Access scenario (see Fig. 3b) MAs are installed in Global and local zones. The MA installed in a local zone is able to perform only operations on the given zone depending on the assigned privileges. The JIMS client - e.g. Client2 in Fig. $3 b$-has to connect to specific JIMS MAs to perform monitoring and management activities.

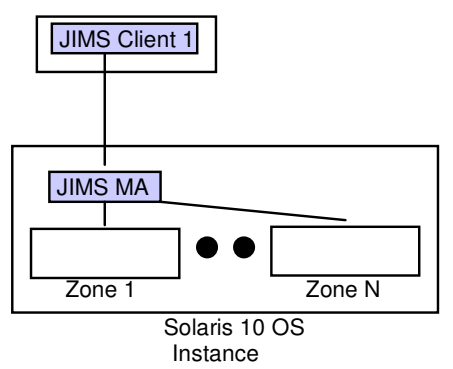

Fig. 3a. "Global Access" deployment sc-enario with only one JIMS MA installed in the global zone

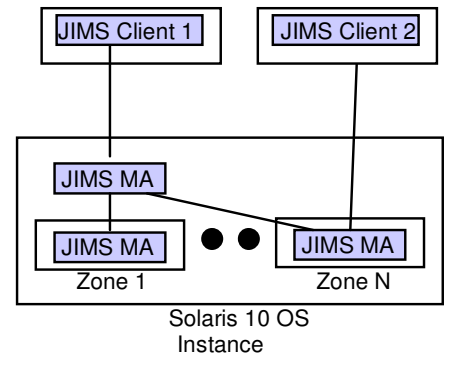

Fig. 3b. "Restricted Access" deployment scen-ario with JIMS MA installed in the global and local zones

The functionality for the monitoring and management of Solaris 10 resources will be exposed via an appropriate API which may be used by some external components. Because JIMS is based on JMX architecture, the MBeans which implement the required functionality 
suit this end perfectly.

\section{JIMS Extension JMX-Based Implementation}

The JIMS extension is a set of MBeans that expose an interface for Solaris 10 resource monitoring and management. These MBeans are divided into three groups: monitoring, management and accounting. Inside each group there are MBeans for zones and projects.

Resource monitoring (Fig. 4) MBeans contain read-only attributes with basic information about zones or projects and their resource usage (CPU, memory, threads etc.) This information is periodically retrieved from /proc VFS with the help of a native library accessed through JNI (files in /proc VFS have platform-dependent binary format and can't be read directly from the Java application).

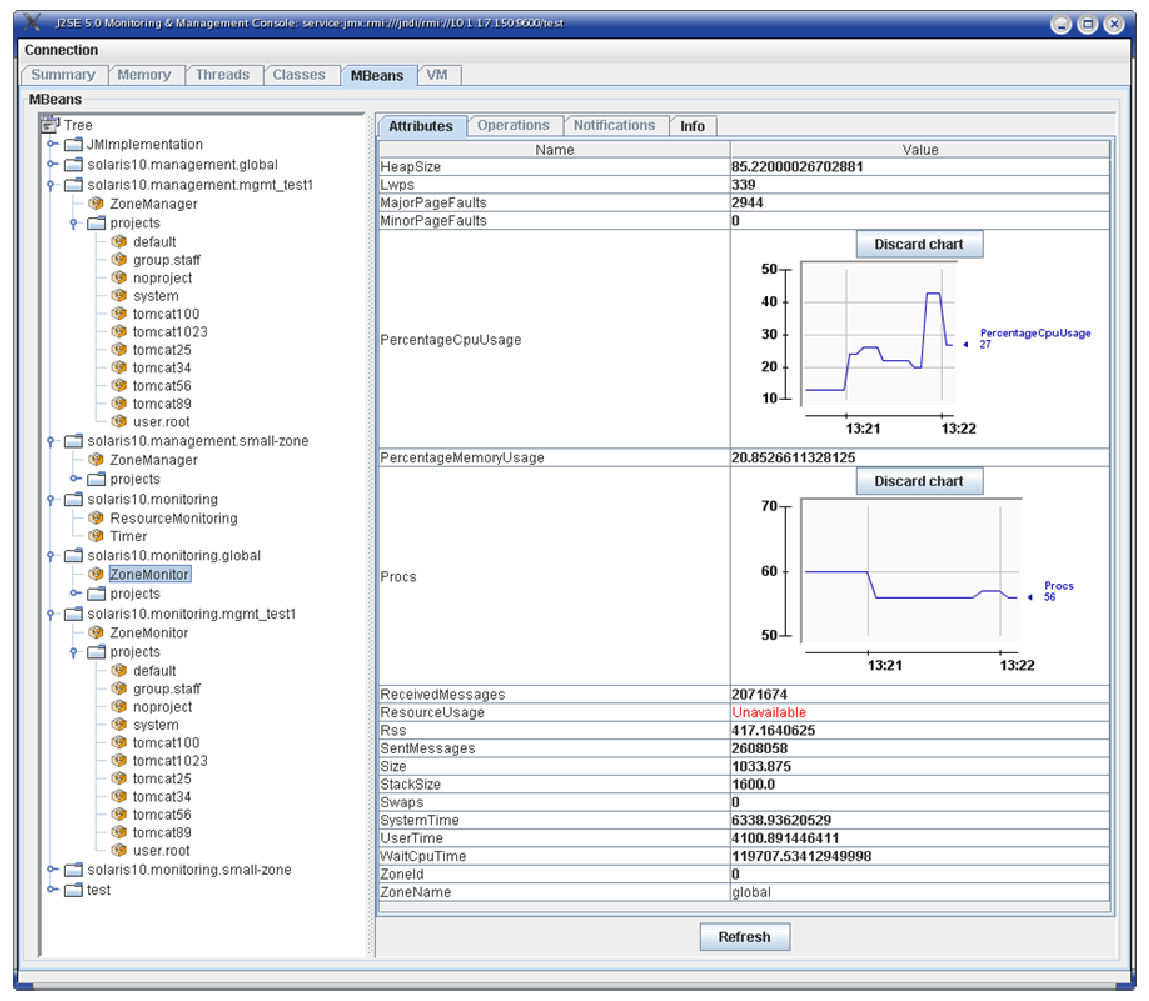

Fig. 4. Solaris 10 resource monitoring MBeans presented in JConsole

Solaris 10 management (Fig. 5) MBeans enable reading and changing properties of zones and projects (i.e. resource controls, member users etc.) and also operations that change zone state. They also allow creating or removing zones and projects. MBean 
interfaces depend on the zone in which the JIMS MA is running (different for global and local zones), i.e. local zone management MBeans expose the boot operation only in the global zone. Management MBeans use various methods to interact with the OS: to collect information about zones and projects, MBeans read configuration files or use JNI. Changes in configuration are applied by executing shell scripts and system commands (with Runtime.exec() invocation). MBeans are able also to emit JMX notifications to inform interested parties about changes in the system (i.e. concerning added projects or changed resource usage).

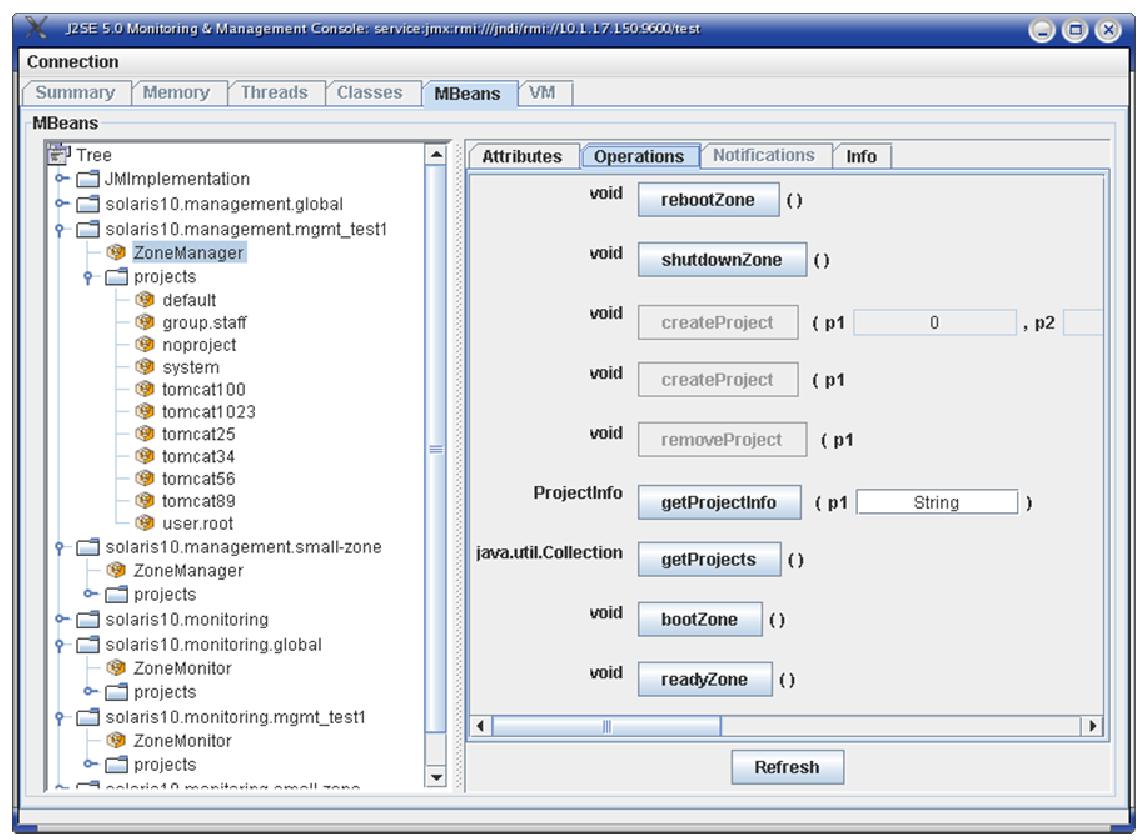

Fig. 5. Solaris 10 management MBeans accessible via JConsole

The accounting group of MBeans exposes long-time historical resource usage information. This information is collected periodically from extended accounting log files and stored in a database. We have decided to use a Java-based DBMS and integrate it within our application (to limit performance overhead). Log files are switched periodically to avoid unnecessary duplicate processing of the same information. Logs can be read only with the use of the libexacct API, available only for $\mathrm{C}$ and Perl (not Java), therefore MBeans access it through a native library and JNI.

\section{Conclusions}

The proposed extension of the JIMS monitoring system with selected Solaris 10 resource monitoring and management mechanisms creates a very coherent and easy to use environment. The provided functionality can be automatically discovered by the 
JIMS self-configuration protocol, following which it may be accessed via a standard web browser using http adapter support, by Web Services (with the JIMS SOAP gateway implementation) or directly by any application written in Java using the standard RMI protocol. The diversity of potential access channels makes the proposed extensions easy to integrate with adaptation layers implemented in any technology.

The proposed concept of JMX-based OS mechanism exposition is rather general and can be applied to any operating system. Therefore the proposed solution can be used for adaptable reflective middleware construction, requiring access to underlying OS functionality. It is also useful for any resource-aware applications or services.

An important feature of the proposed extension is the ability to use various mechanisms for collecting operational data from the operating system. The constructed MBeans exploit polling as well as notification mechanisms, which can reduce operational overhead. The proposed solution can be also easily customized or modified, even at runtime, due to dynamic MBean technology usage.

Acknowledgment. This research has been partially supported by Polish Ministry of Education and Science grant no.1583/T11/2005/29.

\section{References}

1. K. Balos: JIMS -the JMX Infrastructure Monitoring System, http://www.eucrossgrid.org/Seminars-INP/JIMS_monitoring_system.zip

2. K. Balos, D. Radziszowski, P. Rzepa, K. Zielinski, S. Zielinski, ”Monitoring GRID Resources - JMX in Action”, TASK Quarterly, pp. 487-501, 2004

3. Sun Microsystems: Java Management Extension Reference Implementation (JMX), http://java.sun.com/products/JavaManagement/

4. Sun Microsystems: JMX Remote API Specification (JMX Remote API), http://developer.java.sun.com/developer/earlyAccess/jmx/

5. N1 Provisioning System, http://wwws.sun.com/software/products/service_provisioning/ index.html

6. Autonomic Computing, http://www.research.ibm.com/autonomic/

7. Daniel Price, Andrew Tucker "Solaris Zones: Operating System Support for Consolidating Commercial Workloads" (http://www.sun.com/bigadmin/content/zones/zones_lisa.pdf)

8. Amy Rich "Spotlight on Solaris Zones Feature" (http://www.sun.com/bigadmin/features/ articles/solaris_zones.html)

9. Menno Lageman "Solaris Containers - What They Are and How to Use Them"

10. "System Administration Guide: Solaris Containers-Resource Management and Solaris Zones" (http://docs.sun.com/app/docs/doc/817-1592)

11. OpenSolaris project website (http://opensolaris.org) 